\title{
Etude Hydrogéochimique des Eaux Souterraines dans la Formation Gréseuse de la Région de Berberati en République Centrafricaine
}

\author{
E. Foto, \\ C. L. Djebebe Ndjinguim, \\ N. Zoudamba, \\ E. Basse Keke, \\ J. Mabingui,
}

Laboratoire Hydrosciences Lavoisier, Chaire - UNESCO sur l'eau Université de Bangui, Faculté des Sciences, Bangui (RCA)

Doi: 10.19044/esj.2019.v15n6p487 URL:http://dx.doi.org/10.19044/esj.2019.v15n6p487

\section{Résumé}

La population de la République Centrafricaine est inégalement repartie: les zones ouest et sud montrent une densité de population beaucoup plus haute que la partie Est. Pour parvenir à une meilleure connaissance des ressources en eau en présence, la compréhension des conditions de recharge des formations gréseuses et leur interrelation avec les cours d'eau environnants et leur reservoirs constituent les principales lacunes à combler par ce projet. L'étude porte donc sur l'évaluation des ressources en eaux dans la formation gréseuse de Berberati par l'utilisation des techniques hydrogéochimiques de et l'hydrologie isotopiques.

Mots-clés: Formation gréseuse, Berberati, hydrologie isotopique 


\title{
Hydrogeochemical Study of Groundwater in the Sandstone Formation of the Berberati Region in the Central African Republic
}

\author{
E. Foto, \\ C. L. Djebebe Ndjinguim, \\ N. Zoudamba, \\ E. Basse Keke, \\ J. Mabingui,
}

Laboratoire Hydrosciences Lavoisier, Chaire - UNESCO sur l'eau Université de Bangui, Faculté des Sciences, Bangui (RCA)

\begin{abstract}
The population of the Central African Republic is unevenly distributed: the western and southern zones show a much higher population density than the eastern part. To gain a better understanding of the water resources present, understanding the recharge conditions of grès formations and their interrelation with the surrounding watercourses and reservoirs are the main gaps to be filled by this project. The study therefore focuses on the evaluation of water resources in the Berberati grès formation through the use of hydrogeochemical techniques and isotopic hydrology.
\end{abstract}

Keywords: Grès formation, Berberati, isotope hydrology

\section{Introduction}

La République Centrafricaine située au cœur de l'Afrique a une superficie de $623000 \mathrm{~km} 2$ pour une population d'environ 4000000 d'habitants. En matière de ressource en eau, en dépit du potentiel non négligeable dont dispose la RCA, elle demeure encore très peu connue. La zone d'étude se concentre autour des 3 principales villes de Berberati, Carnot et Gamboula. Cette région se situe à l'ouest de Bangui à proximité de la frontière avec le Cameroun. La préfecture de Mambéré-Kadéi, à l'Ouest de la République Centrafricaine, est l'une de ses 16 préfectures. Elle couvre 30203 $\mathrm{km}^{2}$ de superficie et la population totale de cette région est estimée à 364795 habitants, soit $9 \%$ de la population totale de la RCA, avec 10 habitants $/ \mathrm{km}^{2}$ de densité (recensement de 2003). Sa capitale est Berberati. 


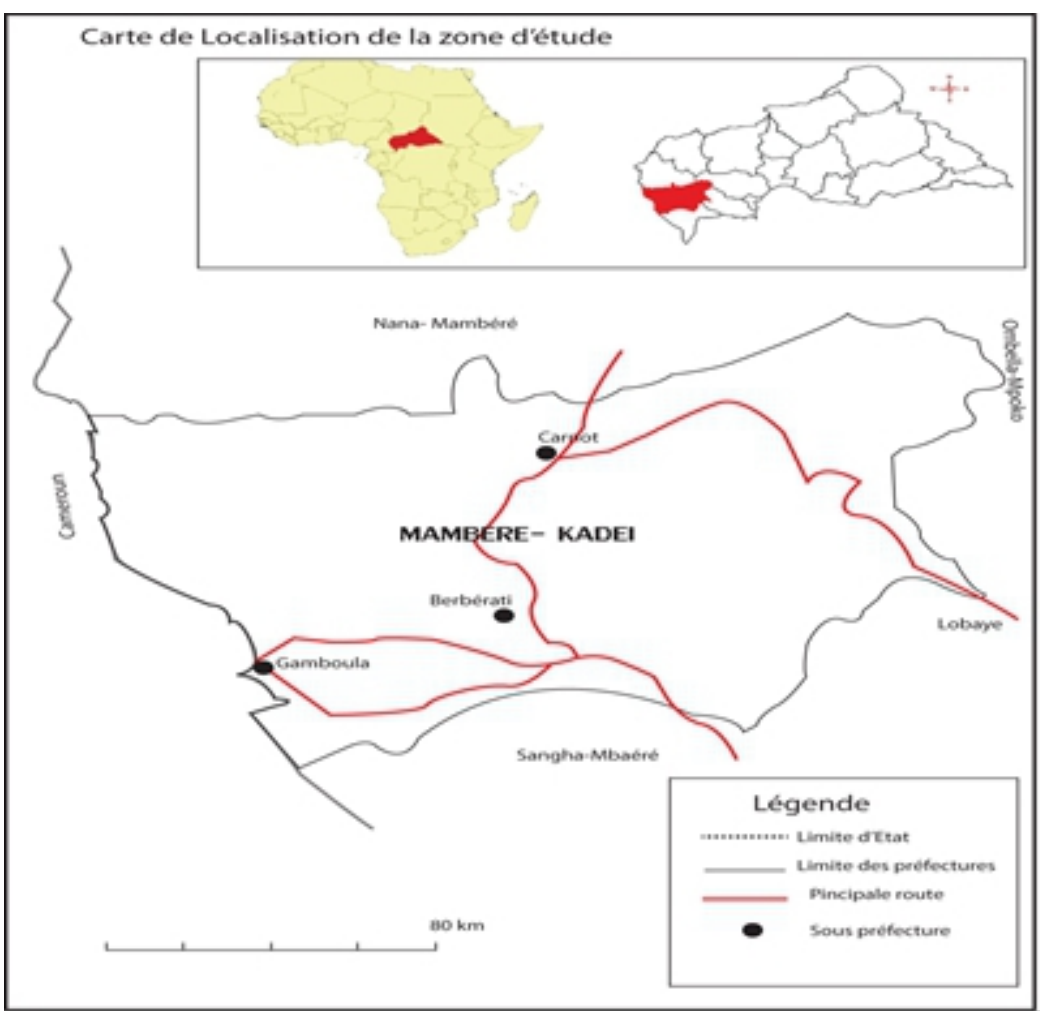

Figure 1 : localisation de la zone d'étude

\section{Cadre Hydrologique et Hydrogéologique}

D'un point de vue hydrologique, la préfecture de Mambéré Kadéi dispose d'un réseau hydrographique très important composé de la Sangha, la Mambéré, la Nana et la Kadéi.

Le relief rencontré est constitué des plateaux d'environ $350 \mathrm{~m}$ d'altitude. Les sols qu'on y rencontre sont de types ferralitiques rouges et ocre, des sols ferralitiques à hydromorphe de profondeur, sols hydromorphes des plaines alluviales, (savane sols rocheux, cuirassés ou gravillonnaires, sols ferralitiques bien drainés.

Concernant l'hydrogéologie, on distingue dans la préfecture de Mambéré Kadéi, des Formations gréseuses mésozoïques de Carnot et de Berberati, qui constituent de par leur perméabilité, leur épaisseur, leur extension et leur situation géographique, dans la zone bien alimentée par les pluies, des aquifères continus susceptibles de renfermer des ressources bien renouvelées, et pour l'instant pratiquement inexploités, si ce n'est pour l'hydraulique villageoise. Bien qu'il existe dans les grès des intercalations argileuses, la nappe est généralement libre, comme les forages dans les grès de Carnot l'ont montré. La profondeur du niveau statique, de l'ordre de 30 à 40 mètres représente cependant un handicap pour le creusement de puits 
traditionnels. Le drainage des aquifères se fait par les rivières, telles la Lobaye et la Mambéré dans les grès de Carnot et Berbérati. On peut toutefois observer de nombreuses sources à débit faible ou moyen.

Grossièrement horizontaux et métamorphiques, les grès de Carnot reposent sur les assises granitiques ou cristalophyllliennes du socle. Les plateaux de Carnot/Berberati forment une cuvette, remplie des niveaux conglomératiques d'épaisseur variant de 5 à $80 \mathrm{~m}$ dans lesquels sont localisés les gisements diamantifères de la dite localité (Boulevert. y, 1983).

J.P. WOLFF (1956) a établi la succession des formations géologiques de la manière suivante :

A la base, des niveaux conglomératiques repérés seulement en rive gauche de la Sangha et non signalés dans la région de la Motao.

Au-dessus, un ensemble gréso-quartziteux qui, vers le sommet, passe progressivement aux schistes argileux par séquences oscillantes. Ces schistes argileux peuvent eux-mêmes être surmontés par quelques niveaux de grèsquartzites. Les grès de carnot/Berbérati d'âge crétacé sont des formations transgressives sur la formation glaciaire de Mambérè, elle est discordante sur le complexe schisto-quartzitique au sud et complexe granito-gneissique au Nord. On distingue également la série fluvio-lacustre de Carnot d'épaisseur variant de 100 à $200 \mathrm{~m}$. Elle se compose de : Conglomérats, Grès et siltes. Les formations gréseuses du bassin de Carnot-Gadzi constituent le remplissage d'une grande dépression creusée dans les formations cristallines du socle. L'ensemble du remplissage gréseux secondaire repose sur un paléo relief accidenté, modelé dans une série fluvio-glaciaire à prédominance argileuse et conglomératique. Les ressources hydrauliques emmagasinées dans ce bassin sont considérables ; le débit spécifique de la Lobaye, cours d'eau qui draine profondément le grès de Carnot par des vallées creusées jusqu'au socle cristallin, atteint $9 \mathrm{l} / \mathrm{s} / \mathrm{km} 2$ (ricolvi, 1987). Les formations gréseuses constituent à cet effet, tant par le débit instantané prévisible des captages, que par les ressources disponibles, un aquifère de grande importance (Cornacchia et al, 1990)La nappe gréseuse présente un caractère libre mais le niveau piézométrique est souvent profond (30 à $45 \mathrm{~m}$ dans les grès de Carnot, parfois en dessous de $50 \mathrm{~m}$. Les aquifères sont en communication avec les fleuves qui les drainent; des lignes de sources ont été observées au contact grès-socle.

\section{I.1. Climatologie}

La climatologie de la zone d'étude a été succinctement étudiée dans les années 1950 par Benoit-Janin (1954). Celui-ci à suivi quatre stations climatiques situées à proximité/sur de la zone d'étude : Nola en région de grande forêt, Boda et Carnot en savane et Berbérati à la limite des deux.

Leur climat est caractérisé par l'alternance d'une saison sèche de novembre à mars (seuls décembre et janvier ont une pluviométrie inférieur à 
$30 \mathrm{~mm}$ ) et d'une saison des pluies d'avril à octobre. La pluviométrie annuelle est légèrement inférieure à $1500 \mathrm{~mm}$ pour Boda, Carnot et Nola, et légèrement supérieure à Berberati. On observe une diminution des pluies durant une courte période de 15 à 20 jours aux mois de juin ou juillet, diminution trop faible pour être qualifiée de petite saison sèche. Les courbes de la figure 2 permettent de constater la similitude de répartition des précipitations sur les quatre stations, seule Berbérati s'en distingue par le nombre plus élevé de jours de pluie.

L'humidité relative moyenne demeure élevée toute l'année, à Berberati ses valeurs limites sont $69 \%$ en février et $86 \%$ en août-septembre. Les rosées sont abondantes. Les températures minima moyennes sont de 17 à $20^{\circ} \mathrm{C}$, les températures maxima de 29 à $33{ }^{\circ} \mathrm{C}$ (Berbérati). Le climat se caractérise donc par une saison des pluies abondante avec humidité forte toute l'année et températures à amplitude de variation faible. En limite nord de la zone climatique guinéenne, forestière ou équatoriale, très humide avec une pluviométrie qui avoisine les $1500 \mathrm{~mm}$ par an et une courte saison sèche, la préfecture de la Mambéré Kadéi est une zone de forêt au sud et de savane arbustive au nord.
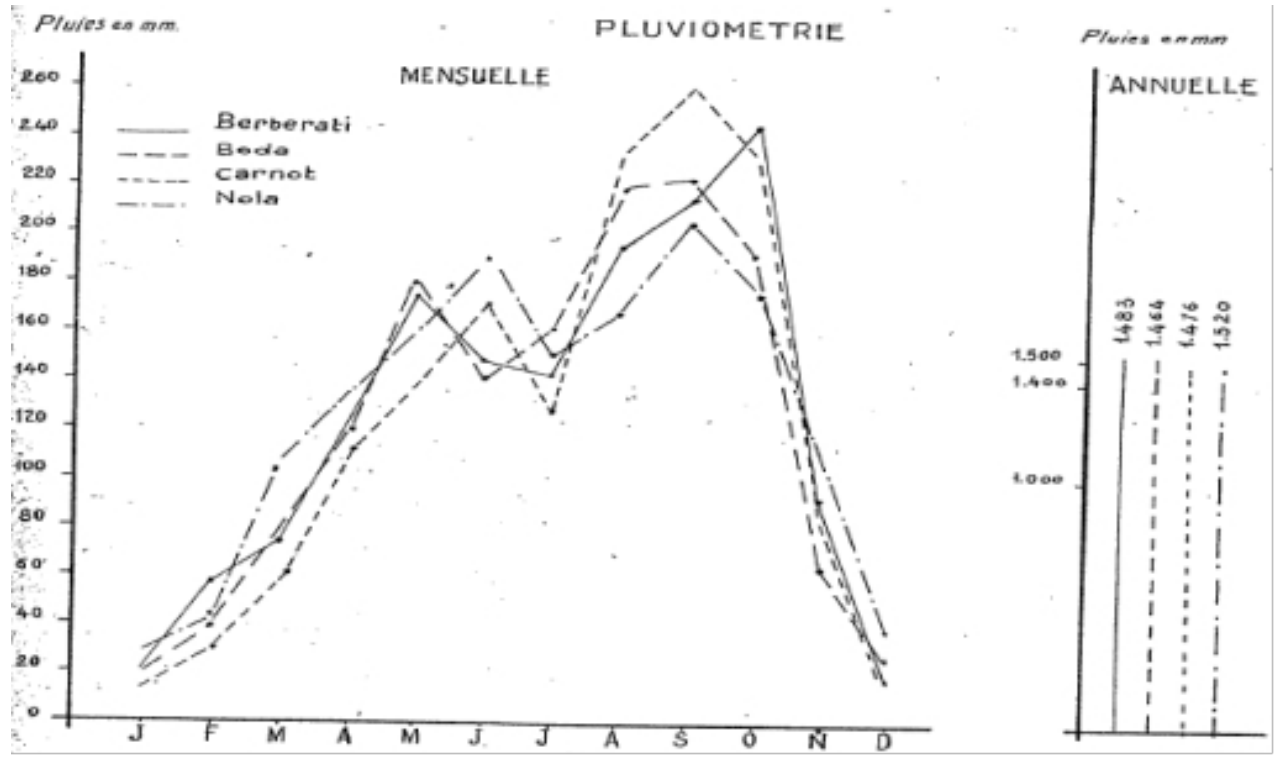

Figure 2 : Pluviométrie enregistrée dans la région de Carnot-Berberati (Benoit-Janin, 1954)pas d'information sur la durée des observations.

\section{I.2. Topographie et hydrographie (Benoit-Janin, 1954)}

L'ensemble du secteur gréseux est un plateau de 600 à $700 \mathrm{~m}$ d'altitude faiblement vallonné mais dans lequel les rivières ont creusé des vallées profondes. Celles-ci appartiennent au système de la Lobaye pour la zone nord, à celui de la Mbaéré et de la Bodengué (affluents de la Lobaye) au sud, à celui de la Mambéré (affluent de la Sangha) à l'ouest. Le réseau hydrographique est 
très peu dense, particulièrement dans le sud où il existe des plateaux de plusieurs milliers d'ha dépourvus de tout point d'eau.

\section{Matériels et Méthodes}

Après une première phase de reconnaissances des ouvrages à prélever sur le terrain par l'équipe du laboratoire, pendant la campagne, 30 points ont été échantillonnés dont forages (25), puits (02), source (02) et eau de rivière(01) au mois juillet en période de hautes eaux.

Sur chaque point d'eau à prélever les analyses in situ suivantes ont été réalisées :

- $\quad$ pH (pH-mètre WTW 340i)

- Conductivité électrique et Température (conductimètre WTW 340i)

- Alcalinité (trousse Alkalinité HACH)

Une série de prélèvements a été réalisés pour différentes analyses chimiques et isotopiques :

- 2 piluliers de $30 \mathrm{ml}$ pour l'analyse du deutérium et de l'oxygène-18,

- 1 flacon 0.51 pour le dosage du tritium sur 10 points,

- 3 flacons de $30 \mathrm{ml}$ filtrés sur $0.45 \mu$ pour le dosage des cations (puis acidification par HNO3), anions et éléments traces (puis acidification par HNO3 ultra-pur) respectivement. Sur chaque forage et puits, les mesures et prélèvements ont été réalisés après avoir renouvelé suffisamment l'eau de la colonne de l'ouvrage lorsque les équipements le permettaient.

Les analyses chimiques et les analyses des isotopes stables de la molécule d'eau ont été réalisées au Laboratoire de Radio-Analyses et Environnement de Tunisie, Les analyses GNIP et GNIR ont été menées à bien dans les laboratoires internes de l'AIEA. Les points de prélèvement ont été sélectionnés afin de couvrir de façon complète l'ensemble de la préfecture de Mambéré Kadéi et en fonction des ouvrages accessibles et disponibles (Figure. 3). 


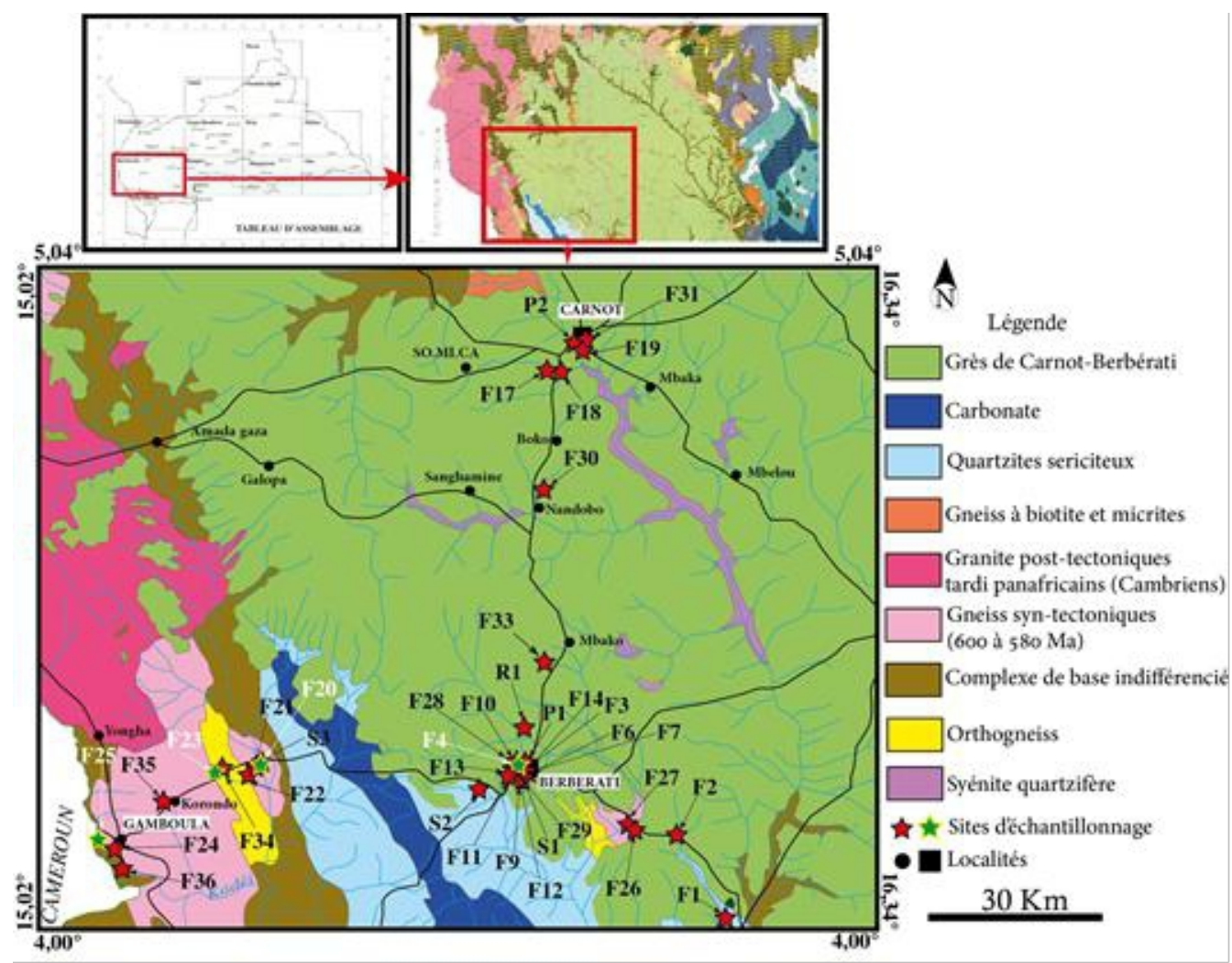

Figure 3 : localisation des points de prélèvement sur la carte géologique de la région.

\section{Résultats et Discussions \\ III.1. Paramètres in situ}

Les valeurs de température des eaux échantillonnées sont comprises entre 23 et $28^{\circ} \mathrm{C}$, avec une moyenne de $26^{\circ} \mathrm{C}$, comparables à la moyenne des températures ambiantes de la région. Dans l'ensemble, les eaux de la zone d'étude ont un $\mathrm{pH}$ variant entre 4,21 et 5,86. Cette acidité est probablement liée à l'hydrolyse des silicates et des acides humiques provenant de la décomposition de la matière organique des zones d'infiltration.Les eaux souterraines de la Mamberé- Kadéi sont faiblement minéralisées, avec des conductivités variant de 8 à $671 \mu \mathrm{S} / \mathrm{cm}$. Les ouvrages ayant des valeurs élevées de conductivités électriques $(200$ à $691 \mu \mathrm{S} / \mathrm{cm})$ sont probablement sujets à contamination anthropique par les latrines confere Tableau 1.

L'alcalinité mesurée sur le terrain reste elle aussi à un niveau faible entre 50 et $100 \mathrm{mg} / \mathrm{l}$ étant donné l'absence de ciment carbonaté dans les grès de Carnot. Les échantillons provenant des granodiorites montrent toutefois des teneurs légèrement plus faibles autour de $80 \mathrm{mg} / \mathrm{l}$.

Globalement les différences dans les paramètres physico-chimiques entre les grès et les granodiorites semblent minimes. 
Tableau 1: resultats des mésures in situ.

\begin{tabular}{|c|c|c|c|c|c|c|c|c|c|c|c|}
\hline $\mathbf{N}^{\circ}$ & $\begin{array}{c}\text { Code } \\
\text { (Nom local) }\end{array}$ & Type & Aquifere & Date & $\begin{array}{c}\text { Latitude } \\
\text { N/S } \\
\text { DDMMSS.DD }\end{array}$ & $\begin{array}{c}\text { Longitude } \\
\text { W/E } \\
\text { DDDMMSS.DD }\end{array}$ & $\begin{array}{c}\text { Altitude } \\
\text { (m) }\end{array}$ & $\begin{array}{c}\text { Cond } \\
\mu \mathrm{S} / \mathrm{cm}\end{array}$ & \begin{tabular}{|c} 
Temp. \\
${ }^{\circ} \mathrm{C}$ \\
\end{tabular} & $\begin{array}{c}\text { pH } \\
\text { units }\end{array}$ & $\begin{array}{c}\text { Alk } \\
\mathrm{mg} / \mathrm{L}\end{array}$ \\
\hline 1 & Bania & $\mathrm{F} 1$ & Grès & $25 / 07 / 2014$ & $04^{\circ} 00^{\prime} 00.4^{\prime \prime}$ & $016^{\circ} 06^{\prime} 54.0^{\prime \prime}$ & 469 & 27 & 26,2 & 4,35 & 3,17 \\
\hline 2 & Gbanbang & $\mathrm{F} 2$ & Grès & $25 / 07 / 2014$ & $04^{\circ} 08^{\prime} 58.6^{\prime \prime}$ & $016^{\circ} 01^{\prime} 75.1^{\prime \prime}$ & 516 & 175 & 26,6 & 3,52 & 10,24 \\
\hline 3 & Ecole Pref. & $\mathrm{F} 3$ & Grès & $26 / 07 / 2014$ & $04^{\circ} 15^{\prime} 48.8^{\prime \prime}$ & $015^{\circ} 47^{\prime} 36.6^{\prime \prime}$ & 571 & 102 & 24,4 & 3,98 & 3,05 \\
\hline 4 & Hopital 1 & $\mathrm{~F} 4$ & Grès & $26 / 07 / 2014$ & $04^{\circ} 15^{\prime} 73.0^{\prime \prime}$ & $015^{\circ} 47^{\prime} 37.1^{\prime \prime}$ & 597 & 47 & 25 & 4,6 & 3,66 \\
\hline 5 & Hopital (D) & F5 & Grès & $26 / 07 / 2014$ & $04^{\circ} 15^{\prime} 79.1^{\prime \prime}$ & $015^{\circ} 47^{\prime} 28.6^{\prime \prime}$ & 589 & 79 & 25,7 & 4,12 & 6,1 \\
\hline 6 & çaval & F6 & Grès & $26 / 07 / 2014$ & $04^{\circ} 15^{\prime} 64.3^{\prime \prime}$ & $015^{\circ} 47^{\prime} 50.7^{\prime \prime}$ & 602 & 56 & 25,4 & 4,09 & 2,68 \\
\hline 7 & çava3 & F7 & Grès & $26 / 07 / 2014$ & $04^{\circ} 15^{\prime} 50.4^{\prime \prime}$ & $015^{\circ} 47^{\prime} 91.4^{\prime \prime}$ & 610 & 39 & 26,2 & 3,69 & 1,22 \\
\hline 8 & Sagbanda & $\mathrm{F} 8$ & Grès & $26 / 07 / 2014$ & $04^{\circ} 15^{\prime} 08.1^{\prime \prime}$ & $015^{\circ} 47^{\prime} 55.2^{\prime \prime}$ & 599 & 50 & 26,8 & 4,56 & 9,15 \\
\hline 9 & Ecole Padrepio & F9 & Grès & $26 / 07 / 2014$ & $04^{\circ} 14^{\prime} .43,6^{\prime \prime}$ & $015^{\circ} 47^{\prime} 48.9^{\prime \prime}$ & 579 & 19 & 25,8 & 4,55 & 5,49 \\
\hline 10 & Source Padrepio & S1 & Grès & $26 / 07 / 2014$ & $04^{\circ} 14^{\prime} 47.1^{\prime \prime}$ & $015^{\circ} 47^{\prime} 57.9^{\prime \prime}$ & 555 & 29 & 25,9 & 4,03 & 2,44 \\
\hline 11 & Paroisse SC & F10 & Grès & $26 / 07 / 2014$ & $04^{\circ} 15^{\prime} 17.6^{\prime \prime}$ & $015^{\circ} 47^{\prime} 34.2^{\prime \prime}$ & 593 & 39 & 26,2 & 4,61 & 5,86 \\
\hline 12 & Gbassola2 & F11 & Grès & $26 / 07 / 2014$ & $04^{\circ} 14^{\prime} 74.1^{\prime \prime}$ & $015^{\circ} 46^{\prime} 96.1^{\prime \prime}$ & 615 & 24 & 26,4 & 4,35 & 5,49 \\
\hline 13 & Deleke(BM) & F12 & Grès & $26 / 07 / 2014$ & $04^{\circ} 15^{\prime} 01.6^{\prime \prime}$ & $015^{\circ} 46^{\prime} 59.8^{\prime \prime}$ & 618 & 52 & 26,3 & 3,85 & 2,2 \\
\hline 14 & Belle-vue & F13 & Grès & $26 / 07 / 2014$ & $04^{\circ} 14^{\prime} 89.1^{\prime \prime}$ & $015^{\circ} 46^{\prime} 08.5^{\prime \prime}$ & 599 & 52 & 26,5 & 3,83 & 2,56 \\
\hline 15 & Ecole 29 mars & $\mathrm{P} 1$ & Grès & $26 / 07 / 2014$ & $04^{\circ} 15^{\prime} 28.0^{\prime \prime}$ & $015^{\circ} 46^{\prime} 89.8^{\prime \prime}$ & 589 & 120 & 26,9 & 4,39 & 6,34 \\
\hline 16 & Potopoto & F14 & Grès & $26 / 07 / 2014$ & $04^{\circ} 16^{\prime} 23.9^{\prime \prime}$ & $015^{\circ} 47^{\prime} 04.6^{\prime \prime}$ & 568 & 671 & 27 & 3,07 & 0 \\
\hline 17 & Nandobo & F15 & Grès & $27 / 07 / 2014$ & $04^{\circ} 39^{\prime} 71.9^{\prime \prime}$ & $015^{\circ} 47^{\prime} 84.0^{\prime \prime}$ & 554 & 26 & 27,4 & 3,82 & 1,708 \\
\hline 18 & Nandobo2 & F16 & Grès & $27 / 07 / 2014$ & $04^{\circ} 41^{\prime} 32.2^{\prime \prime}$ & $015^{\circ} 48^{\prime} 70.1^{\prime \prime}$ & 600 & 71 & 26,5 & 5,21 & 35,38 \\
\hline 19 & Ngogbara & F17 & Grès & $27 / 07 / 2014$ & $04^{\circ} 53^{\prime} 68.5^{\prime \prime}$ & $015^{\circ} 49^{\prime} 28.8^{\prime \prime}$ & 551 & 31 & 28 & 3,33 & 2,318 \\
\hline 20 & Kouisso & F18 & Grès & $27 / 07 / 2014$ & $04^{\circ} 53^{\prime} 58.7^{\prime \prime}$ & $015^{\circ} 50^{\prime} 42.9^{\prime \prime}$ & 497 & 8 & 27,1 & 4,63 & 3,294 \\
\hline
\end{tabular}




\begin{tabular}{|c|c|c|c|c|c|c|c|c|c|c|c|}
\hline 21 & EEB & F19 & Grès & $27 / 07 / 2014$ & $04^{\circ} 56^{\prime} 36.7^{\prime \prime}$ & $015^{\circ} 52^{\prime} 76.9^{\prime \prime}$ & 561 & 78 & 26,5 & 2,94 & 0 \\
\hline 22 & Camp. Fonct. & $\mathrm{P} 2$ & Grès & $27 / 07 / 2014$ & $04^{\circ} 56^{\prime} 21.2^{\prime \prime}$ & $015^{\circ} 52^{\prime} 21.3^{\prime \prime}$ & 505 & 253 & 26,6 & 3,27 & 3,05 \\
\hline 23 & Batouri & $\mathrm{R} 1$ & Grès & 28/07/2014 & $04^{\circ} 18^{\prime} 04.0^{\prime \prime}$ & $015^{\circ} 48^{\prime} 38.0^{\prime \prime}$ & 514 & 26 & 23,4 & 4,99 & 8,3 \\
\hline 24 & Source Xavier & $\mathrm{S} 2$ & Grès & 29/07/2014 & $04^{\circ} 13^{\prime} 67.2^{\prime \prime}$ & $015^{\circ} 43^{\prime} 05.5^{\prime \prime}$ & 572 & 21 & 24,8 & 4,21 & 8,66 \\
\hline 25 & Nassole & $\mathrm{F} 20$ & Granodiorite & $29 / 07 / 2014$ & $04^{\circ} 15^{\prime} 58.5^{\prime \prime}$ & $015^{\circ} 22^{\prime} 69.8^{\prime \prime}$ & 534 & 197 & 25,6 & 5,73 & 64,66 \\
\hline 26 & Nangoko & $\mathrm{F} 21$ & Granodiorite & 29/07/2014 & $04^{\circ} 15^{\prime} 11.2^{\prime \prime}$ & $015^{\circ} 21^{\prime} 48.3^{\prime \prime}$ & 541 & 81 & 25,1 & 5,33 & 122 \\
\hline 27 & Doumba & $\mathrm{F} 22$ & Granodiorite & 29/07/2014 & $04^{\circ} 14^{\prime} 99.4^{\prime \prime}$ & $015^{\circ} 21^{\prime} 06.0^{\prime \prime}$ & 550 & 85 & 25,3 & 5,24 & 48,07 \\
\hline 28 & Soroma & $\mathrm{F} 23$ & Granodiorite & $29 / 07 / 2014$ & $04^{\circ} 14^{\prime} 38.6^{\prime \prime}$ & $015^{\circ} 18^{\prime} 37.4^{\prime \prime}$ & 555 & 113 & 25,5 & 5,36 & 70,52 \\
\hline 29 & Centre de santé & $\mathrm{F} 24$ & Granodiorite & 29/07/2014 & $04^{\circ} 07^{\prime} 23.5^{\prime \prime}$ & $015^{\circ} 08^{\prime} 30.1^{\prime \prime}$ & 616 & 68 & 26 & 5,22 & 26,352 \\
\hline 30 & Douane & $\mathrm{F} 25$ & Granodiorite & 30/07/2014 & $04^{\circ} 08^{\prime} 15.5^{\prime \prime}$ & $015^{\circ} 06^{\prime} 79.9^{\prime \prime}$ & 602 & 198 & 25,6 & 5,86 & 107,36 \\
\hline
\end{tabular}

\section{III.2. Faciès chimique des eaux :}

\section{- Ions majeurs}

Les analyses des ions majeurs ne montrent pas de ségrégation forte entre les grès de Carnot et le substratum cristallin fait de granodiorites. La chimie des anions est dominée par les bicarbonates, chlorures et nitrates ( confere Annexe1 : Données physicochimiques).

Les teneurs moyennes des cations des eaux analysées présentent l'ordre d'abondance suivant: $\mathrm{Ca}:^{2+}>\mathrm{Na}^{+}>\mathrm{K}^{+}>$ $\mathrm{Mg}^{2+}$. Pour les anions, l'ordre d'abondance est : $\mathrm{NO}_{3}{ }^{-}>\mathrm{HCO}_{3}{ }^{-}>\mathrm{Cl}^{-}>\mathrm{SO}_{4}{ }^{2-}$, De ce qui suit, l'essentiel de la minéralisation de nos eaux est la dominance en ions nitrates. On note également une grande dispersion de la silice dans les eaux.

Les concentrations en potassium et magnesium relativement fortes sont à relier avec l'hydrolyse des mineraux feldspathiques connus dans les grès. 


\section{- Diagramme de Piper}

\section{Diagramme de Piper}

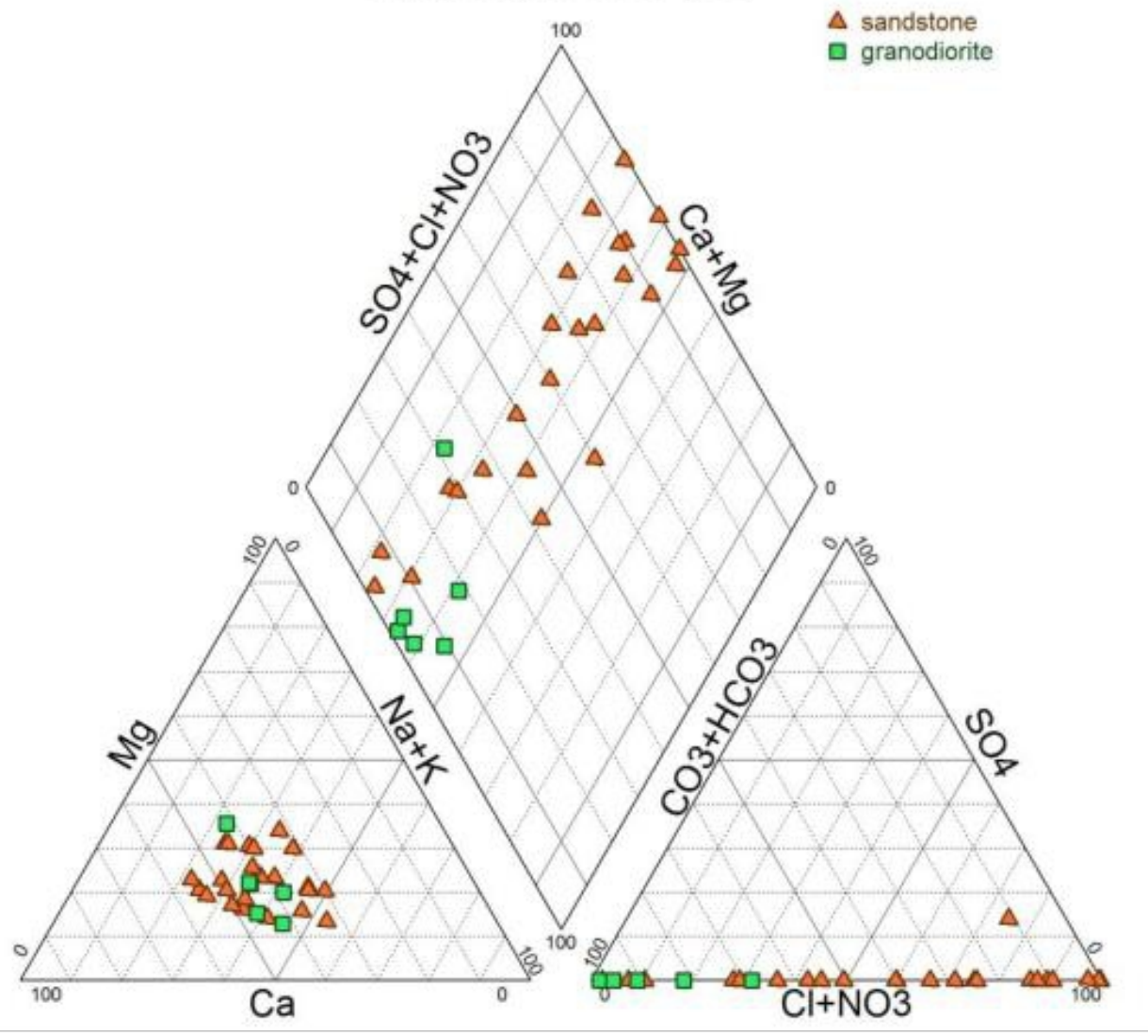

Figure 4 : diagramme de Piper des les eaux souterraines de la région de Mambéré Kadéi.

La représentation de nos échantillons pour la période sèche dans le diagramme de Piper (figure 4) à partir des données des ions majeurs permet d'identifier deux principaux types de faciès chimiques pour les eaux souterraines de la région de la Mambéré-Kadéi:

- les eaux chlorurées nitratées(forages superficiels et puits traditionnels); et

- les eaux bicarbonatées calciques et magnésiens (forages profonds et sources) d'une part,

\section{- Diagramme de Stiff}

Les diagrammes de Stiff obtenus à partir des échantillons d'eau souterraine (figure 5) ont permis de diviser les eaux en 3 groupes présentant :

- une forte minéralisation : Les forages gréseux bicarbonaté (F4, F20, F23, F25...); les puits, en général marquent de fortes pollutions par les nitrates et sulfates 
- une faible minéralisation pour les forages superficiels

- les sources, très faiblement minéralisées.
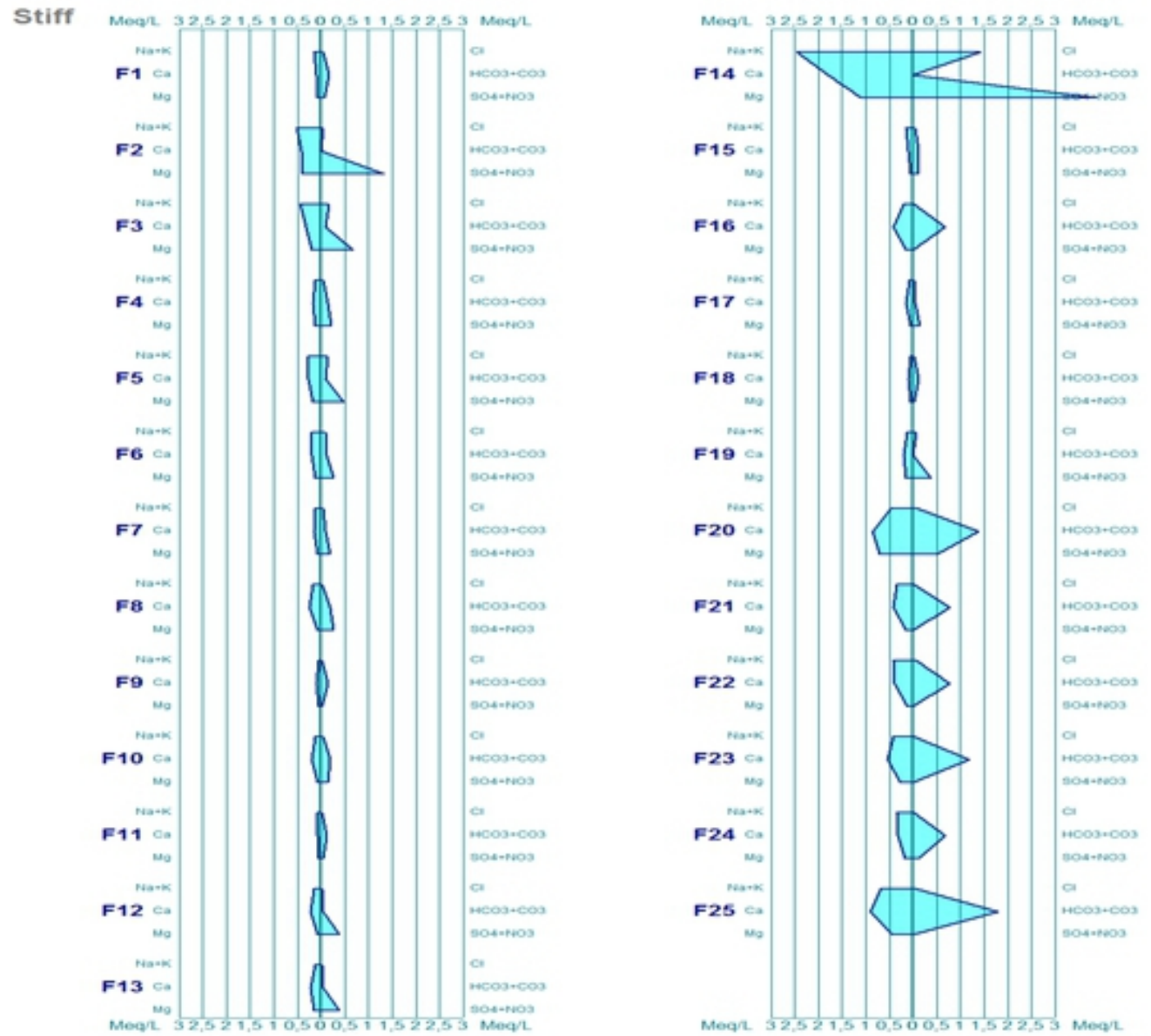

Puits Sources et Rivière

Stiff

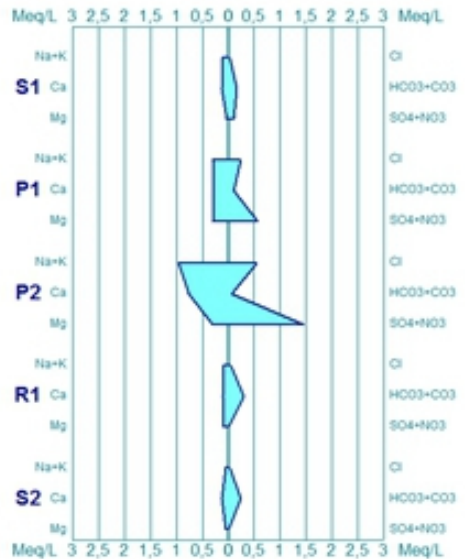

Figure 5 : diagramme de Stiff, minéralisation dans les eaux de la Mambéré Kadéi 


\section{III.4 Influences anthropiques sur l'aquifère}

Les activités humaines liées au lavage, à la cuisine ou encore les décharges d'immondices plus ou moins présentes sur le territoire ont une influence notable sur la qualité des eaux souterraines les plus superficielles( tableau 1 en annexe 2). Une très forte corrélation peut ainsi être notée entre les teneurs en chlorures et les teneurs en sodium et potassium sur les eaux des puits (Figure6 ). Les eaux des forages présentent quant à elles des teneurs beaucoup plus faibles proches des apports atmosphériques et terrigènes attendus dans la région.

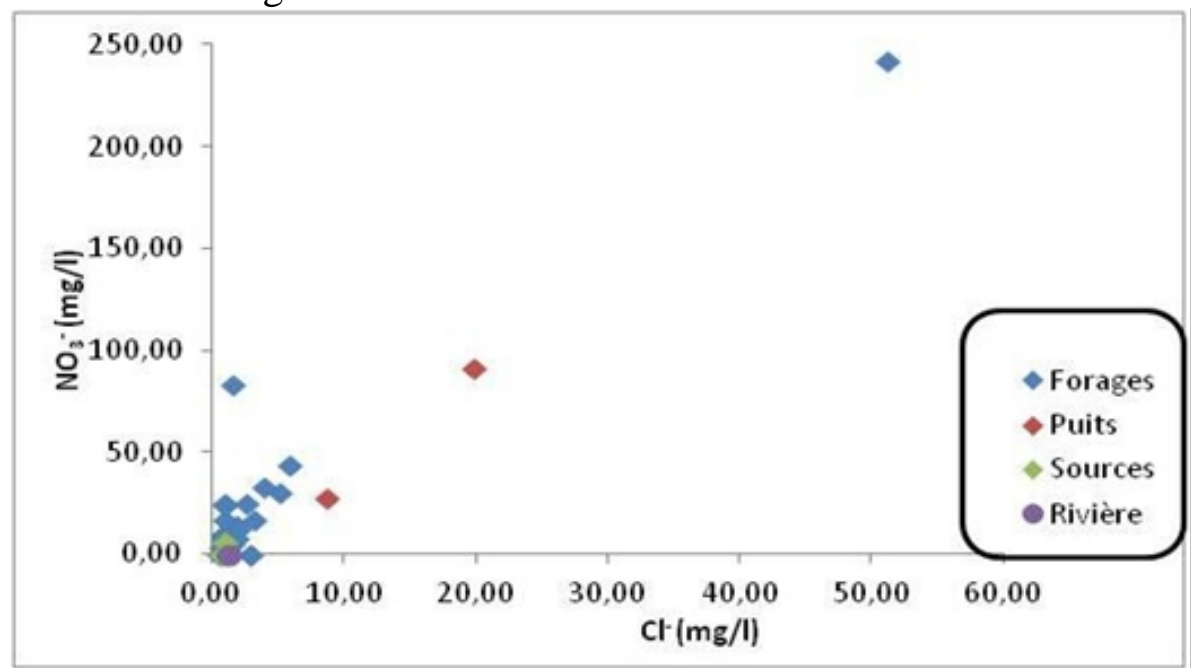

Figure 6 : Corrélation entre les ions Nitrates et chlorure dans les eaux souterraines.

Dans le Tableau 2 ci -dessous, on constate une forte corrélation entre les chlorures et les sels totaux dissous(TDS) ainsi que la conductivité, ceci montre bien leur participation à la minéralisation. Il en est de même pour les nitrates et chlorures ainsi que nitrate et sodium prouvant ainsi l'origine anthranpique des pollutions. 
Tableau 2: Matrice de correlation des eaux echantillonnées

\begin{tabular}{|c|c|c|c|c|c|c|c|c|c|c|c|c|c|c|c|c|}
\hline & TDS & $\mathrm{pH}$ & $\mathrm{c} 25^{\circ} \mathrm{C}$ & $\mathrm{Ca}$ & $\mathrm{Mg}$ & $\mathrm{Na}$ & $\mathrm{K}$ & NH4 & $\mathrm{H} 3 \mathrm{O}+$ & $\mathrm{HCO} 3$ & $\mathrm{CO} 3$ & $\mathrm{Cl}$ & $\mathrm{SO} 4$ & PO4 & NO3 & $\mathrm{NO} 2$ \\
\hline TDS & 1 & & & & & & & & & & & & & & & \\
\hline $\mathrm{pH}$ & $-0,49$ & 1 & & & & & & & & & & & & & & \\
\hline $\mathrm{c} 25^{\circ} \mathrm{C}$ & 0,99 & $-0,54$ & 1 & & & & & & & & & & & & & \\
\hline $\mathrm{Ca}$ & 0,99 & $-0,4$ & 0,96 & $39,40 \%$ & & & & & & & & & & & & \\
\hline $\mathrm{Mg}$ & 0,95 & $-0,42$ & 0,94 & 0,94 & $22,40 \%$ & & & & & & & & & & & \\
\hline $\mathrm{Na}$ & 0,98 & $-0,55$ & 0,99 & 0,95 & 0,9 & $25,80 \%$ & & & & & & & & & & \\
\hline $\mathrm{K}$ & 0,93 & $-0,56$ & 0,95 & 0,89 & 0,8 & 0,96 & $12,00 \%$ & & & & & & & & & \\
\hline NH4 & 0 & 0 & 0 & 0 & 0 & 0 & 0 & $0,00 \%$ & & & & & & & & \\
\hline $\mathrm{H} 3 \mathrm{O}+$ & 0,81 & $-0,71$ & 0,85 & 0,72 & 0,75 & 0,84 & 0,81 & 0 & $0,40 \%$ & & & & & & & \\
\hline $\mathrm{HCO} 3$ & 0,23 & 0,4 & 0,11 & 0,36 & 0,3 & 0,12 & $-0,01$ & 0 & $-0,21$ & $38,90 \%$ & & & & & & \\
\hline $\mathrm{CO} 3$ & 0 & 0 & 0 & 0 & 0 & 0 & 0 & 0 & 0 & 0 & $0,40 \%$ & & & & & \\
\hline $\mathrm{Cl}$ & \begin{tabular}{|l|}
0,89 \\
\end{tabular} & $-0,58$ & 0,93 & $\mathbf{0 , 8 3}$ & 0,79 & 0,93 & 0,95 & 0 & $\mathbf{0 , 8 1}$ & $-0,17$ & 0 & $14,20 \%$ & & & & \\
\hline $\mathrm{SO} 4$ & 0 & 0 & 0 & 0 & 0 & 0 & 0 & 0 & 0 & 0 & 0 & 0 & $0,00 \%$ & & & \\
\hline $\mathrm{PO} 4$ & 0 & 0 & 0 & 0 & 0 & 0 & 0 & 0 & 0 & 0 & 0 & 0 & 0 & $0,00 \%$ & & \\
\hline $\mathrm{NO} 3$ & 0,87 & $-0,7$ & 0,92 & 0,79 & 0,81 & 0,9 & 0,91 & 0 & 0,92 & $-0,27$ & 0 & 0,94 & 0 & 0 & $46,40 \%$ & \\
\hline $\mathrm{NO} 2$ & 0 & 0 & 0 & 0 & 0 & 0 & 0 & 0 & 0 & 0 & 0 & 0 & 0 & 0 & 0 & $0,00 \%$ \\
\hline
\end{tabular}




\section{III.5 Modélisation géochimique}

Un modèle simple du programme géochimique PREEQC est utilisé afin de déterminer les Indices de saturations des minéraux qui sont à l'origine de la minéralisation des eaux souterraines de Mambéré Kadéi, en relation avec les terrains encaissants. Les resultats sont reportés sur la figure 7 ci-dessous.

Un indice SI supérieur à zéro précise que l'eau souterraine est sursaturée par rapport à la matière minérale particulière et elle est donc incapable de dissoudre plusieurs minéraux. Une telle valeur de l'indice reflète les eaux souterraines de décharge d'un aquifère contenant une quantité suffisante de la matière minérale avec suffisamment de temps de séjour pour atteindre l'équilibre. Néanmoins, la sursaturation peut également être produite par d'autres facteurs qui comprennent la dissolution incongruante, l'effet d'ion commun, l'évaporation, l'augmentation rapide de la température et de la concentration en CO2 dissous (Langmuir, 1997 ;Ako et al., 2010). Pour la plupart de nos échantillons, on assiste donc à une sursaturation en Chalcédoine et quartz, en relation avec la nature des formations géologique du secteur d'étude. Nous avons vu que les formations de Carnot-Berberati sont gréseuse, à ciment siliceux, celle de Gamboula, granodioritique, contiennent toutes des quartz. L'hypothèse de l'hydrolyse des silicates pourrait être évoquée à cet effet dans l'aquifère de Carnot Berberati.
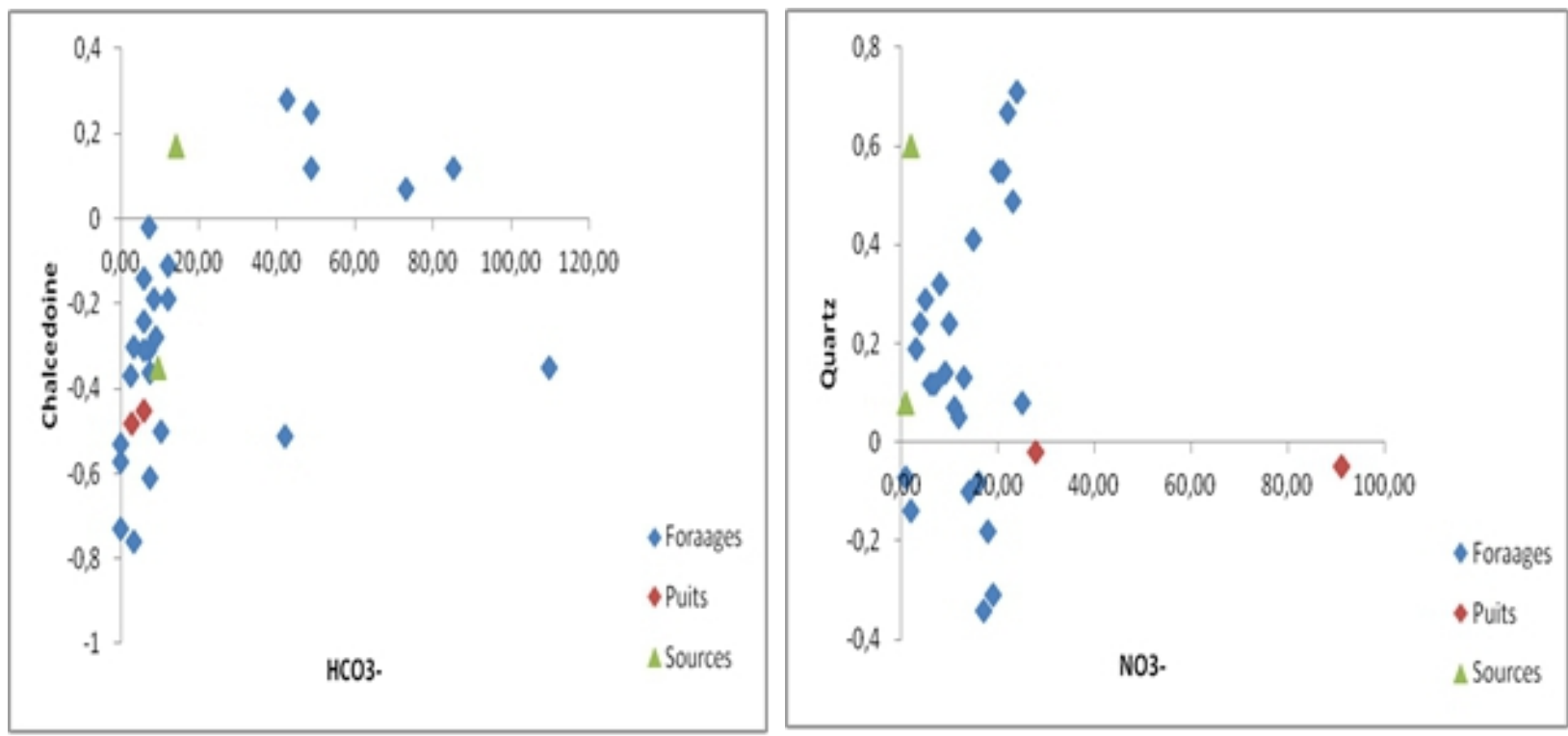


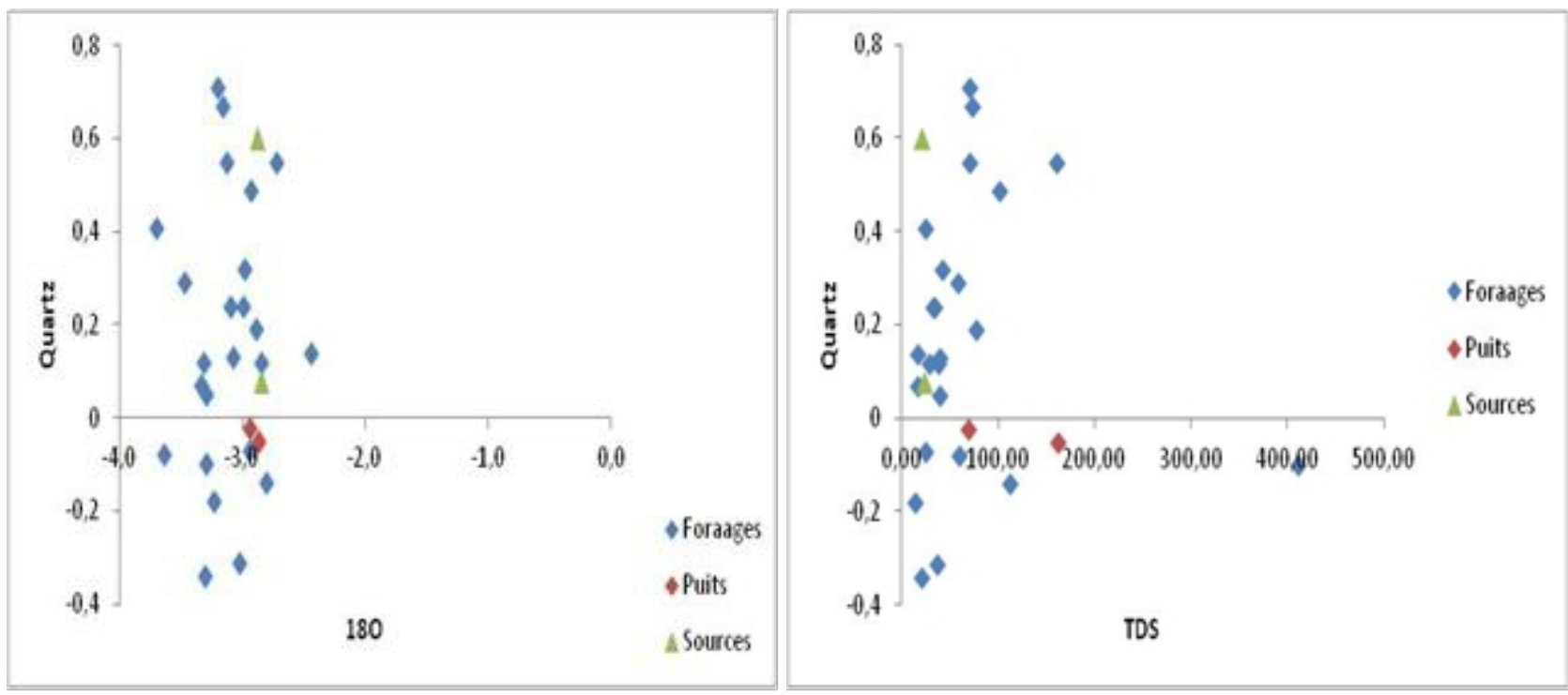

Figure 7 : Indice de saturation en certains éléments dans les eaux souterraines de Mambéré Kadéi.

\section{III.6 Analyses isotopiques}

Il n'existe pas de différence notable entre les échantillons provenant des grès et ceux provenant des roches cristallines du substratum. La chronique de données ne montre pas d'influence notable de l'évaporation et les excès en deutérium sont compris entre 9 et $11 \%$.

Les teneurs en tritium sont notables dans l'ensemble des échantillons analysés avec des valeurs comprises entre 2 et 3 UT, ce qui est conforme à la teneur actuelle des pluies dans cette région du monde (Djebebe-Ndjiguim, 2014). Pour deux points F22 (Doumba) et F25 (Douane) voir Tableau 3, on observe des activités plus faible autour de 1 UT qui pourraient traduire un mélange entre des eaux récentes d'origine météorique et des eaux souterraines plus profondes et plus anciennes. Il est à noter que ces deux points correspondent à des puits forés dans les granodiorites.

La signature isotopique des eaux de l'aquifère des grès de Carnot semble donc assez homogène et traduit une origine récente des eaux. Néanmoins, il convient de bien noter que les ouvrages échantillonnés lors de cette étude sont des ouvrages superficiels qui n'interceptent que les 100 premiers mètres de l'aquifère au maximum, ce qui est marginal compte tenu d'une puissance de $400 \mathrm{~m}$ pour cet aquifère dans sa partie centrale. Il est donc parfaitement imaginable de trouver des eaux beaucoup plus anciennes dans les niveaux les plus profonds de l'aquifère, pour peu que des ouvrages capables de les intercepter soient réalisés. 
Tableau 3: Résultats des analyses Isotopiques.

\begin{tabular}{|c|c|c|c|}
\hline Sample Name & $\delta^{2} H$ (\%o vs Smow) & $\delta^{18} \mathrm{O}(\%$ vs Smow) & ${ }^{3} \mathbf{H}(\mathrm{TU})$ \\
\hline Bania & $-11,3$ & $-2,9$ & \\
\hline Gbanbang & $-13,1$ & $-2,8$ & 1,79 \\
\hline Ecole pref & $-14,9$ & $-2,9$ & \\
\hline Hopital1 & $-12,8$ & $-3,0$ & \\
\hline Hopital D & $-15,5$ & $-3,5$ & \\
\hline Cava1(F6) & $-15,3$ & $-3,3$ & 2,67 \\
\hline Cava3 & $-13,4$ & $-2,9$ & \\
\hline Sagbanda & $-14,2$ & $-3,0$ & \\
\hline Ecole padrepio & $-11,5$ & $-2,4$ & \\
\hline Source padrepio & $-13,4$ & $-2,8$ & 2,91 \\
\hline Paroisse sc & $-16,6$ & $-3,1$ & \\
\hline Gbassola2 & $-15,0$ & $-3,3$ & \\
\hline Deleke BM & $-15,1$ & $-3,3$ & \\
\hline Belle vue & $-12,9$ & $-3,1$ & \\
\hline Ecole 29 mars & $-14,4$ & $-2,9$ & 2,29 \\
\hline Potopoto & $-14,0$ & $-3,3$ & 2,62 \\
\hline Nandobo & $-16,3$ & $-3,7$ & \\
\hline Nandobo2 & $-15,9$ & $-3,6$ & \\
\hline Ngogbara & $-14,3$ & $-3,3$ & \\
\hline Kouisso & $-17,1$ & $-3,2$ & \\
\hline EEB & $-15,4$ & $-3,0$ & \\
\hline Camp fonctionn. & $-13,1$ & $-2,9$ & 3,08 \\
\hline Batouri & $-12,4$ & $-3,0$ & \\
\hline Source Xavier & $-13,1$ & $-2,9$ & \\
\hline Nassole & $-13,9$ & $-3,1$ & 2,11 \\
\hline Nangoko & $-15,0$ & $-2,7$ & \\
\hline Doumba (F22) & $-16,3$ & $-3,2$ & 1,31 \\
\hline Soroma & $-14,4$ & $-2,9$ & \\
\hline Centre de santé & $-15,3$ & $-3,2$ & 2,88 \\
\hline Douane (F23) & $-16,5$ & $-3,2$ & 0,86 \\
\hline
\end{tabular}




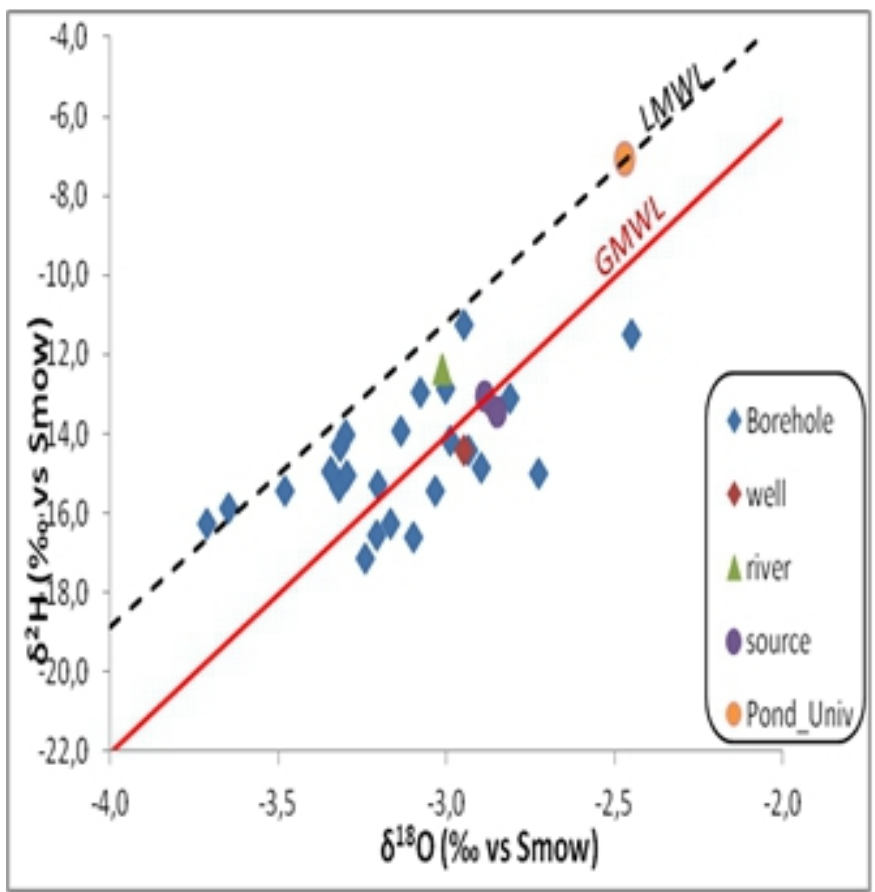

Figure 8: Relation ${ }^{18} \mathrm{O}$ et ${ }^{2} \mathrm{H}$ dans les eaux souterraines de Mambéré Kadéi

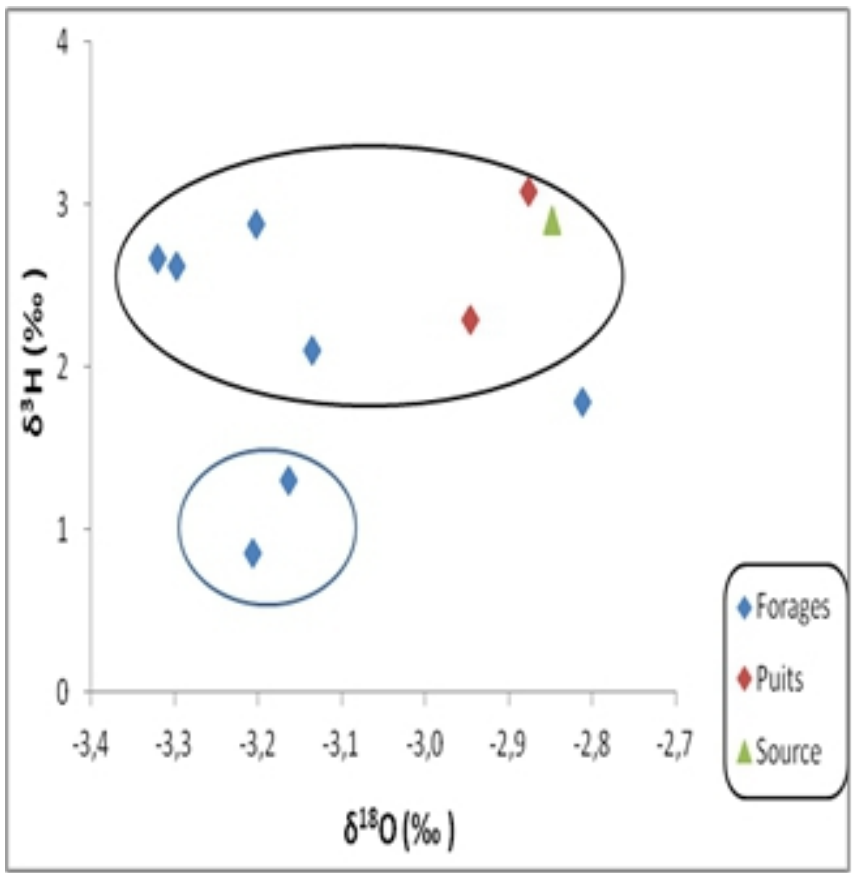

Figure 9: Corrélation ${ }^{3} \mathrm{H}$ vs $\delta^{18} \mathrm{O}$ dans les eaux souterraines de Mambéré Kadéi

\section{Conclusion}

Ce travail a montré que la région présente un certain nombre d'enjeux socio-économique autour de l'eau. Elle constitue une première démarche 
d'investigations sur les eaux souterraines comme ressource de substitution aux eaux de surface anthrophisées.

Il a permis d'identifier deux principaux types de faciès chimiques pour les eaux souterraines de la région de la Mambéré-Kadéi, les eaux chlorurées nitratées (forages superficiels et puits traditionnels); et les eaux bicarbonatées calciques et magnésiens (forages profonds, sources) d'une part. Les eaux minéralisées provenant des forages gréseux bicarbonatés (F4, F20, F23, F25...) ; des puits, en général marquent de fortes pollutions par les nitrates et sulfates. Les eaux faiblement minéralisées que sont les forages superficiels. La modélisation géochimique indique une sursaturation en Chalcédoine et quartz, dans les eaux, confirme la nature gréseuse (Carnot Berberati) et granodiritique (Gamboula) des formations géologiques du secteur d'étude.

Remerciements : Les auteurs remercient tout le personnel du Laboratoire Hydrosciences de l'Université de Bangui pour les campagnes d'échantillonnage et l'Agence Internationale de l'Energie Atomique (AIEA ainsi que le Laboratoire de Radio-Analyses et Environnement de Tunisie pour avoir réalisé les analyses chimiques et les isotopes stables de la molécule d'eau.

\section{References :}

1. Alvarez, P., 1995. Evidence of a Neoproterozoïc carbonate ramp on the northern edge of the Central African craton: relation with Late Proterozoïc intracratonic troughs. Geologische Rundschau, 84, 636648.

2. Alvarez, P., 1997. Morphologies karstiques et implications minières en République Centrafricaine. Journal of African Earth Sciences, 25, 293-305.

3. Boulvert, Y. and Juberthie, C., 1998. République Centrafricaine. In:

C. Juberthie and V. Decu, eds. Encyclopaedia biospeologica.Moulis: Société de Biospéologie, 1659-1668.

4. Boulvert, Y. and Salomon, J.N., 1988. Sur l'existence de paléocryptokarsts dans le basin de l'Oubangui (République Centrafricaine). Karstologia, 11-12, 37-48.

5. Cornacchia, M., Detay, M., and Giorgi, L., 1990. Nouvelles données sur l'hydrogéologie centrafricaine. Hydrogéologie, 3, 165-181.

6. Djebebe-Ndjiguim, CL, 2007. Application des modèles hydrogéochimiques sur le bassin versant de Bangui. [Application of hydro-geochemical models to the Bangui catchment.] Master,Thesis, Univ. Bangui, 77p.

7. Djebebe-Ndjiguim C.L., Huneau F., Denis A. , Foto E., Moloto-aKenguemba G., Celle Jeanton H. , Garel E. , Jaunat J., Mabingui J.and 
Le Coustumer P., Characterization of the aquifers of the Bangui urban area, Central African Republic, as an alternative drinking water supply resource.Hydrological Sciences Journal $\mathrm{N}^{\circ} 58$ (8) pp : 17601778.2013

8. Kangadjaba , E., Impact des Peocessus Hydrochimique sur la Qualité des eaux souterraines dans la ville de Ndjamena - Tchad, European Scientifi Journal, vol 14, june 2018

9. Ketchemen B., Etude hydrogéologique du grand Yaéré (Extrême-nord Cameroun) Synthèse hydrogéologique et étude de la recharge par les isotopes de l'environnement. Thèse de l'Université Cheikh Anta Diop de Dakar, 216 p. 1992.

10. Poidevin, J.L., 1976. Les formations du Précambrien supérieur de la Région de Bangui (République Centrafricaine). Bulletin Société Géologique France, 18, 999-1003.

11. Poidevin, J.L., 1996. Un segment proximal de rampe carbonate d'âge protérozoique supérieur au Nord du craton d'Afrique centrale (sud-est de la République Centrafricaine). Journal of African Earth Sciences, 23, 257-262.

12. Runge, J \& Nguimalet, CR, 2005. Physiogeographic features of the Oubangui catchment and environmental trends reflected in discharge and floods at Bangui 1911-1999, Central African Republic. Geomorphology 70, 311-324

13. Van Der Wal, A, 2009. "Connaissances des méthodes de captage des eaux souterraines appliquées aux forages manuels". ["Methods for harnessing groundwater applied to manual drilling."] Fondation Practica, The Netherlands.

14. Wesselink, A, 1996.Les régimes hydroclimatiques et Hydrogéologiques d'un bassin versant de type tropical humide: L'Oubangui(RCA).

15. Yidana, SM and al, 2010. Analysis of groundwater quality using multivariate and spatial analyses in the Keta basin, Ghana. Journal of African Earth Sciences. 
Annexe 1 : Données Physicochimiques

\begin{tabular}{|c|c|c|c|c|c|c|c|c|c|c|c|c|c|c|}
\hline $\mathrm{N}^{\circ}$ & Nom des points d'eau & Date & $\begin{array}{l}\mathrm{CO}_{3}{ }^{2-} \\
\mathrm{mg} / \mathrm{l}\end{array}$ & $\begin{array}{c}\mathrm{HCO}_{3}^{-} \\
\mathrm{mg} / \mathrm{l}\end{array}$ & $\begin{array}{c}\mathrm{Cl}^{-} \\
\mathrm{mg} / \mathrm{l}\end{array}$ & $\begin{array}{l}\mathrm{NO}_{3}^{-} \\
\mathrm{mg} / \mathrm{l}\end{array}$ & $\begin{array}{l}\mathrm{SO}_{4}{ }^{2-} \\
\mathrm{mg} / \mathrm{l}\end{array}$ & $\begin{array}{l}\mathrm{Na}^{+} \\
\mathrm{mg} / \mathrm{l}\end{array}$ & $\begin{array}{c}\mathrm{K}^{+} \\
\mathrm{mg} / \mathbf{l}\end{array}$ & $\begin{array}{l}\mathrm{Ca}^{2+} \\
\mathrm{mg} / \mathrm{l}\end{array}$ & $\begin{array}{l}\mathrm{Mg}^{2+} \\
\mathrm{mg} / \mathrm{l}\end{array}$ & $\begin{array}{l}\text { TDS } \\
\mathrm{mg} / \mathrm{l}\end{array}$ & $\begin{array}{c}\mathrm{Fe} \\
\mathrm{mg} / \mathrm{l}\end{array}$ & $\begin{array}{l}\mathrm{SiO}_{2} \\
\mathrm{mg} / \mathrm{l}\end{array}$ \\
\hline 1 & Bania & $25 / 07 / 2014$ & $<0,1$ & 10,50 & 1,63 & 5,18 & 0,02 & 1,99 & 2,96 & 1,97 & 0,81 & 25,14 & $<0,02$ & 5,36 \\
\hline 2 & Gbanbang & 25/07/2014 & $<0,1$ & $<0,1$ & 1,64 & 83,54 & $<0,02$ & 8,92 & 4,91 & 8,50 & 4,86 & 112,46 & $<0,02$ & 4,55 \\
\hline 3 & Ecole Pref. & 26/07/2014 & $<0,1$ & 6,10 & 5,88 & 43,52 & $<0,02$ & 6,98 & 5,62 & 6,47 & 2,51 & 77,17 & $<0,02$ & 9,81 \\
\hline 4 & Hopital 1 & $26 / 07 / 2014$ & $<0,1$ & 8,50 & 1,93 & 14,13 & $<0,02$ & 1,72 & 2,19 & 3,54 & 1,63 & 33,74 & $<0,02$ & 10,96 \\
\hline 5 & Hopital (D) & 26/07/2014 & $<0,1$ & 6,10 & 5,16 & 30,72 & $<0,02$ & 4,19 & 3,85 & 5,66 & 2,06 & 57,85 & $<0,02$ & 12,31 \\
\hline 6 & çava1 & 26/07/2014 & $<0,1$ & 7,20 & 3,21 & 16,96 & $<0,02$ & 2,48 & 3,14 & 3,80 & 1,40 & 38,29 & $<0,02$ & 8,35 \\
\hline 7 & çava3 & $26 / 07 / 2014$ & $<0,1$ & 6,10 & 2,00 & 12,45 & $<0,02$ & 1,93 & 1,57 & 2,87 & 0,99 & 28,01 & $<0,02$ & 8,33 \\
\hline 8 & Sagbanda & $26 / 07 / 2014$ & $<0,1$ & 12,20 & 1,02 & 17,07 & $<0,02$ & 1,19 & 4,90 & 4,80 & 0,98 & 42,26 & $<0,02$ & 13,21 \\
\hline 9 & Ecole Padrepio & $26 / 07 / 2014$ & $<0,1$ & 9,20 & 1,08 & 1,81 & $<0,02$ & 0,59 & 1,43 & 2,18 & 0,60 & 16,99 & $<0,02$ & 8,76 \\
\hline 10 & Source Padrepio & $26 / 07 / 2014$ & $<0,1$ & 9,50 & 1,04 & 6,28 & $<0,02$ & 1,50 & 2,22 & 2,63 & 0,50 & 23,77 & $<0,02$ & 7,56 \\
\hline 11 & Paroisse SC & $26 / 07 / 2014$ & $<0,1$ & 12,20 & 1,38 & 9,98 & $<0,02$ & 1,19 & 3,24 & 4,07 & 0,84 & 32,99 & $<0,02$ & 10,84 \\
\hline 12 & Gbassola2 & $26 / 07 / 2014$ & $<0,1$ & 7,50 & 0,66 & 3,36 & $<0,02$ & 0,68 & 1,49 & 1,74 & 0,64 & 16,17 & $<0,02$ & 7,35 \\
\hline 13 & Deleke(BM) & $26 / 07 / 2014$ & $<0,1$ & 2,60 & 1,08 & 25,11 & $<0,02$ & 2,40 & 2,19 & 4,32 & 1,04 & 38,84 & $<0,02$ & 7,16 \\
\hline 14 & Belle-vue & $26 / 07 / 2014$ & $<0,1$ & 3,50 & 1,08 & 24,26 & $<0,02$ & 1,82 & 1,81 & 4,52 & 1,92 & 39,02 & $<0,02$ & 8,44 \\
\hline 15 & Ecole 29mars & $26 / 07 / 2014$ & $<0,1$ & 6,10 & 8,81 & 27,88 & 6,26 & 4,50 & 4,96 & 6,14 & 3,91 & 68,55 & $<0,02$ & 6,04 \\
\hline 16 & Potopoto & $26 / 07 / 2014$ & $<0,1$ & $<0,1$ & 51,24 & 242,40 & 0,50 & 42,02 & 25,57 & 35,89 & 13,26 & 410,86 & $<0,02$ & 4,95 \\
\hline 17 & Nandobo & $27 / 07 / 2014$ & $<0,1$ & 7,20 & 1,85 & 7,93 & $<0,02$ & 2,43 & 2,20 & 2,02 & 0,50 & 24,21 & $<0,02$ & 16,19 \\
\hline 18 & Nandobo2 & $27 / 07 / 2014$ & $<0,1$ & 42,20 & 0,55 & $<0,02$ & $<0,02$ & 1,79 & 4,33 & 8,35 & 1,90 & 59,32 & $<0,02$ & 5,21 \\
\hline 19 & Ngogbara & 27/07/2014 & $<0,1$ & 3,50 & 0,92 & 9,98 & $<0,02$ & 1,08 & 0,93 & 2,83 & 0,61 & 19,95 & $<0,02$ & 2,91 \\
\hline 20 & Kouisso & $27 / 07 / 2014$ & $<0,1$ & 7,50 & 0,84 & 1,74 & $<0,02$ & 0,74 & 0,91 & 1,81 & 0,46 & 14,10 & $<0,02$ & 4,19 \\
\hline
\end{tabular}




\begin{tabular}{|c|c|c|c|c|c|c|c|c|c|c|c|c|c|c|}
\hline 21 & EEB & $27 / 07 / 2014$ & $<0,1$ & $<0,1$ & 2,65 & 25,25 & $<0,02$ & 1,96 & 1,25 & 4,04 & 1,73 & 36,97 & $<0,02$ & 3,11 \\
\hline 22 & Camp. Fonct. & $27 / 07 / 2014$ & $<0,1$ & 3,00 & 19,87 & 91,08 & 0,20 & 15,11 & 12,78 & 15,46 & 3,95 & 161,45 & $<0,02$ & 5,57 \\
\hline 23 & Batouri & $8 / 07 / 2014$ & $<0,1$ & 18,30 & 1,27 & $<0,02$ & $<0,02$ & 1,27 & 1,79 & 2,59 & 1,18 & 26,60 & $<0,02$ & 2,06 \\
\hline 24 & Xavier & $29 / 07 / 2014$ & $<0,1$ & 14,20 & 0,67 & $<0,02$ & $<0,02$ & 0,66 & 1,14 & 2,89 & 0,73 & 20,48 & $<0,02$ & 24,90 \\
\hline 25 & & 29/07/2014 & $<0,1$ & 85,40 & 3,95 & 33,21 & $<0,02$ & 9,13 & 2,66 & 17,08 & 8,78 & 160,29 & $<0,02$ & 22,50 \\
\hline 26 & goko & $29 / 07 / 2014$ & $<0,1$ & 48,80 & 1,02 & $<0,02$ & $<0,02$ & 5,55 & 4,52 & 8,45 & 1,70 & 70,23 & $<0,02$ & 22,37 \\
\hline 27 & & 29/07/2014 & $<0,1$ & 48,80 & 2,87 & $<0,02$ & $<0,02$ & 7,77 & 3,80 & 8,22 & 1,51 & 73,16 & $<0,02$ & 29,70 \\
\hline 28 & & $29 / 07 / 2014$ & $<0,1$ & 73,20 & 0,78 & $<0,02$ & $<0,02$ & 6,94 & 5,16 & 11,17 & 3,39 & 100,83 & $<0,02$ & 19,69 \\
\hline 29 & entre d & $29 / 07 / 2014$ & $<0,1$ & 42,70 & 0,93 & 8,08 & $<0,02$ & 5,87 & 3,70 & 6,50 & 2,04 & 69,92 & $<0,02$ & 32,16 \\
\hline 30 & Douane & $30 / 07 / 2014$ & $<0,1$ & 109,80 & 2,95 & $<0,02$ & $<0,02$ & 12,30 & 6,34 & 18,10 & 5,54 & 155,23 & $<0,02$ & 7,55 \\
\hline
\end{tabular}

